\title{
Reflexión, Rumiación Negativa y Desarrollo de Sintomatología Depresiva en Adolescentes de Sexo Femenino
}

\author{
Reflection, Negative Rumination and Development of Depressive Symptomatology \\ in Female Adolescents
}

\author{
Félix Cova S.* Paulina Rincón G. \\ Universidad de Concepción, Chile \\ Roberto Melipillán A. \\ Universidad del Desarrollo, Chile \\ (Rec: 24 de marzo 2009 Acep: 21 de septiembre 2009)
}

\begin{abstract}
Resumen
Diversas investigaciones han observado que la rumiación se asocia a consecuencias negativas en el bienestar y salud mental, y que podría ser uno de los factores que contribuya al ánimo y trastornos depresivos en las adolescentes. Recientemente se ha propuesto que el concepto habitualmente empleado de rumiación abarca al menos dos dimensiones distintas, la reflexión y la rumiación negativa. Sería esta última la más perjudicial. La presente investigación evalúa, de forma prospectiva, la influencia de la reflexión y la rumiación negativa en el desarrollo de sintomatología depresiva en una muestra de 268 adolescentes de sexo femenino. La primera medición se realizó cuando éstas cursaban primero medio; la segunda medición corresponde a la obtenida en el seguimiento de un año. Los resultados confirman que la rumiación negativa es efectivamente un factor de riesgo de desarrollo de síntomas depresivos. También se confirmó que la reflexión no muestra ninguna asociación significativa en términos prospectivos con estos últimos, sólo transversal. Estos resultados corroboran la utilidad de delimitar mejor el concepto de rumiación e indican que sería conveniente que las estrategias destinadas a prevenir y tratar la depresión en adolescentes aborden la existencia de patrones rumiativos negativos.
\end{abstract}

Palabras claves: Pensamiento repetitivo, reflexión, rumiación, depresión, ansiedad, adolescencia.

\begin{abstract}
Different studies have found that rumination is associated with negative consequences for wellbeing and mental health. This indicates that rumination could be one of the factors that contribute to mood and depressive disorders in female adolescents. Recently it has been suggested that the concept of rumination includes at least two different dimensions: reflection and negative rumination. The latter would be the most harmful. This research assessed, in a prospective basis, the influence of reflection and negative rumination $\mathrm{n}$ the development of depressive symptoms in a sample of 268 female adolescents. The first measurement was undertaken when the group was attending their first grade of secondary education and this assessment was repeated a year later. The results confirm that negative rumination is indeed a risk factor for development of depressive symptoms. It was also confirmed that the reflection showed no significant prospective associations but only an association with transactional analyses. These results confirm the need of refining the concept of rumination and the value of addressing the negative ruminative patterns when defining the strategies to prevent and treat depression in adolescents
\end{abstract}

Key words: Repetitive thought, reflection, brooding, rumination, depression, anxiety, adolescent. 


\section{Introducción}

En las últimas décadas, desde diversas tradiciones de investigación, se ha generado interés por delimitar la existencia de formas de pensamiento repetitivas y/o persistentes, y por evaluar sus efectos en las personas. Watkins (2008) señala como ejemplos de conceptos que se han propuesto y que pueden ser englobados dentro de la noción de formas repetitivas de pensamiento, a la preocupación, rumiación, cognición perseverativa, procesamiento emocional, procesamiento cognitivo, simulación mental, reflexión, resolución de problemas, entre otros.

Entre los conceptos que aluden a formas de pensamiento repetitivo, uno de los que más relevancia ha tenido en la investigación ha sido el de rumiación (Papageorgiou \& Siegle, 2003). Aun cuando han existido diversas formas de conceptualizar la rumiación, la que ha tenido más difusión la describe como el pensamiento pasivo y repetitivo sobre el malestar anímico y depresión que se estén experimentando, y sobre sus posibles causas y consecuencias (NolenHoeksema, 2004). De allí que esta forma de conceptualizar la rumiación reciba también el nombre de rumiación depresiva (Papageorgiou \& Wells, 2004).

La Teoría del Estilo de Respuesta, propuesta originalmente por Nolen-Hoeksema (1991), sostiene, entre otros aspectos, que este pensamiento pasivo, repetitivo y focalizado en los propios estados de tristeza y abatimiento contribuye a la severidad y mantención del ánimo y trastornos depresivos. Esta teoría ha estimulado mucha de la investigación relativa a la rumiación y sus efectos. En concordancia con sus supuestos, se ha observado que la rumiación es un modo de afrontamiento y regulación emocional que tiene cierta estabilidad en el tiempo; estudios experimentales han confirmado que la rumiación exacerba negativamente tanto el ánimo como la memoria autobiográfica y dificulta la resolución de problemas (Lyubomirsky \& Nolen-Hoeksema, 1995; Lyubomirsky, Caldwell, \& Nolen-Hoeksema, 1998); estudios transversales y longitudinales han corroborado la relación de la rumiación con el desarrollo de síntomas y trastornos depresivos y ansiosos en población general y también en adolescentes (Abela \& Hankin, 2009, Cova, Rincón \& Melipillán, 2007; Kuyken, Watkins, Holden \& Cook, 2006). Asimismo, existe evidencia creciente de la relación de la rumiación con otros problemas psicopatológicos aparte de la depresión, aunque la investigación al respecto ha sido más escasa (Nolen-Hoeksema, Stice, Wade \& Bohon, 2007; Thomsen, 2006).

Se ha planteado que los efectos negativos de la rumiación tendrían relación con el amplificar los pensamientos negativos, interrumpir el procesamiento para la resolución de problemas, interferir el afrontamiento activo, mantener y potenciar los estresores negativos, y disminuir en el largo plazo el apoyo social (Nolen-Hoeksema, 2004; Papageorgiou \& Wells, 2004).
El instrumento desarrollado por Nolen-Hoeksema, denominado Escala de Respuestas Rumiativas (RRS), ha tenido gran importancia en la investigación en el tema. Este instrumento era originalmente una medida de autoinforme de 22 ítems, referido a pensamientos y conductas que se realizan al estar triste o "bajoneado". Posteriormente Treynor, González y Nolen-Hoeksema (2003) modificaron este instrumento, dado que 12 de sus 22 ítemes tenían cierta superposición con la sintomatología depresiva y podrían contribuir a resultados equívocos. El análisis de factores principales de esta nueva versión depurada del instrumento permitió identificar dos subfactores de rumiación: reflection y brooding. El primer concepto, traducible como reflexión, se refiere a conductas que tienen relación con el análisis de las dificultades vividas ("voy a algún lugar solitario para pensar acerca de mis sentimientos", "analizo mi personalidad para tratar de comprender por qué estoy deprimido"). El concepto de brooding no es fácil de traducir, porque su significado es equivalente al de rumiación. Sería una forma específica de ésta que implica un pensamiento con valencia emocional negativa (queja) focalizado en el malestar experimentado y que se asocia a una evaluación negativa de sí mismo (“¿qué he hecho para merecer esto?”, “ipor qué tengo problemas que otras personas no tienen?"). En una investigación reciente, brooding fue traducido al español como mascullamiento (Cova et al., 2007), sin embargo, esta denominación también se presta a confusión. En el presente artículo se le denominará "rumiación negativa".

La importancia de la distinción entre estos dos factores es que permite ir clarificando qué aspectos de los pensamientos repetitivos, y de la rumiación en particular, pueden ser particularmente negativos, y cuáles pueden ser eventualmente útiles o positivos. Pensar sobre las experiencias $\mathrm{y}$ vivencias negativas no es necesariamente perjudicial $\mathrm{y}$, de hecho, hay evidencia que ciertas formas de focalización en los sentimientos y experiencias dolorosas pueden ser positivas y necesarias (Watkins, 2008). Es de importancia, en consecuencia, delimitar la noción de rumiación e identificar los procesos específicos que implica para poder dar cuenta de estas contradicciones aparentes.

De hecho, el estudio de Treynor et al. (2003) encontró que la reflexión y la rumiación negativa se comportaron diferencialmente respecto a su influencia en el desarrollo de sintomatología depresiva: la reflexión, si bien apareció concurrentemente relacionada con la presencia de sintomatología depresiva, en el tiempo se asoció a una disminución de ésta; en cambio, la rumiación negativa, tanto en forma concurrente como longitudinal, se asoció a la presencia de más sintomatología depresiva. Ello sugiere que la mayor reflexión sobre uno mismo y los problemas que se viven puede ser una respuesta a la presencia de sintomatología depresiva, y no es necesariamente desadaptativa; en cambio, la sólo focalización en el malestar acompañada de evaluación 
y crítica hacia sí mismo parece incrementar la presencia de estados de ánimo disfóricos.

Un estudio reciente encontró respaldo, a través de análisis factoriales tanto exploratorios como confirmatorios, a la distinción entre reflexión y rumiación negativas en la traducción que se realizó de la versión breve y depurada del RSS, para su aplicación en lengua española a adolescentes chilenos (Cova et al., 2007). El objetivo de la presente investigación es analizar la validez predictiva de la distinción entre reflexión y rumiación negativa. En particular, se evalúa la influencia de cada uno de estos procesos en el desarrollo de sintomatología depresiva y ansiosa en una muestra de adolescentes chilenos de sexo femenino.

Este estudio se realiza en población adolescente por la relevancia que adquiere la sintomatología emocional en este periodo de la vida. Consistentemente se ha demostrado un incremento acentuado de la sintomatología emocional luego de la pubertad. Es posible que la consolidación de ciertas formas de regulación emocional y afrontamiento desadaptativas en las adolescentes, sea uno de los factores que explique el aumento acentuado de disforia emocional y de trastornos afectivos en esta etapa vital. El incremento de la sintomatología y trastornos afectivos es particularmente agudo en el género femenino, y es de gran relevancia identificar factores que contribuyan a explicar este fenómeno (Zahn-Waxler, Crick, Shirtcliff, \& Woods, 2006). Existen evidencias que la tendencia rumiativa es mayor en las mujeres que en los hombres; de allí que diversos autores planteen que parte de la mayor prevalencia de síntomas y trastornos afectivos en mujeres respecto a los hombres podría estar mediada por la rumiación (Hilt \& Nolen-Hoeksema, 2009; Zahn-Waxler et al., 2006).

\section{Método}

\section{Participantes}

Los análisis fueron realizados con 268 participantes que tenían los datos completos para todas las variables evaluadas en las dos mediciones consideradas. Son estudiantes de sexo femenino de enseñanza media de 7 liceos municipales y particulares subvencionados de la intercomuna ConcepciónTalcahuano. La gran mayoría (72\%) tenía 14 años al momento de la primera evaluación. El presente estudio considera los datos obtenidos en esta primera evaluación y los obtenidos en la evaluación realizada 12 meses después. Las participantes son parte de un estudio de seguimiento que tiene entre sus objetivos evaluar la efectividad de dos programas preventivos de sintomatología depresiva. Como se detalla posteriormente, tanto la participación en los programas preventivos como el puntaje inicial de depresión fueron controlados estadísticamente en los análisis.

\section{Instrumentos}

Inventario de Depresión de Beck segunda edición $(B D I-I I)$. Es un instrumento desarrollado por Beck, Steer y Brown (1996) de amplio uso internacional dadas sus adecuadas propiedades psicométricas (Whisman, Pérez, \& Ramel, 2000). En la presente investigación se empleó la adaptación al español desarrollada por Sanz, Navarro y Vázquez (2003). El BDI-II es un instrumento de veintiún ítem diseñado para evaluar la gravedad de la sintomatología depresiva. Ha demostrado ser útil tanto en población adulta como adolescente. En cada uno de los ítem, la persona tiene que elegir, entre un conjunto de cuatro alternativas ordenadas de menor a mayor gravedad, la frase que mejor describe su estado durante las últimas dos semanas incluyendo el día en que completa el instrumento. Es uno de los instrumentos más utilizados internacionalmente para este propósito dadas sus excelentes propiedades psicométricas, tanto de la versión original inglesa como de sus traducciones, entre éstas, al español (Beck et al., 1996; Sanz et al., 2003). En Chile, el instrumento ha mostrado discriminar en forma efectiva entre población clínica y no consultante (Melipillán, Cova \& Rincón, 2008). En cuanto a la confiabilidad del BDI-II en la presente investigación, se obtuvo un coeficiente alfa de Cronbach de 0,93 en la primera evaluación y de 0,92 en la de seguimiento.

Inventario de Ansiedad de Beck (BAI). Es un instrumento desarrollado por Beck y Steer (1993), que ha recibido amplia aceptación internacional, dadas sus adecuadas propiedades psicométricas (Piotrowski, 1999). En la presente investigación se empleó la adaptación al español desarrollada por Sanz y Navarro (2003). La escala BAI es un instrumento de veintiún ítems. El proceso de adaptación y validación de este instrumento en adolescentes chilenos confirma las adecuadas propiedades psicométricas del instrumento en población adolescente chilena (Cova \& Melipillán, 2007). La confiabilidad observada en el presente estudio para la escala BAI correspondió a un coeficiente alfa de Cronbach de 0,91 en la primera evaluación y 0,92 en la de seguimiento.

Escala de Respuesta Rumiativa (RRS). Corresponde a la adaptación al español de la versión abreviada de la Ruminative Response Scale (Cova et al., 2007). Esta versión excluye todos los ítemes de la original escala RRS que podrían solaparse con sintomatología depresiva. Análisis factoriales y confirmatorios, tanto de la escala original como de la versión abreviada, indican que esta escala está compuesta por dos factores, de 5 ítems cada una: Reflexión y Rumiación Negativa. Siguiendo las recomendaciones de los autores, se crearon 2 ítems adicionales para cada una de subescalas (para Reflexión: Trato de aprender de la experiencia que he vivido, Trato de descubrir por qué me siento así; para Rumiación Negativa: Le doy vueltas en mi mente una y otra vez a las cosas malas que me han pasado, Recuerdo todas las veces en que me pasan cosas malas). Un análisis 
factorial y confirmatorio mostró que la incorporación de estos ítems estaba justificada, por lo que cada subfactor quedó finalmente compuesto por 7 ítemes. El alfa obtenido en la Escala de Reflexión fue de 0,75 en la primera evaluación y de 0,80 en la de seguimiento, y en la de Rumiación Negativa, fue de 0,80 y 0,82 , respectivamente .

En el RRS cada participante debe autoinformar la frecuencia con la que realiza las conductas indicadas por el instrumento en los momentos en que se encuentra triste o "bajoneado". Cada ítem tiene cuatro opciones de respuesta ( 0 = Casi Nunca, $1=$ A Veces, $2=$ Frecuentemente y $3=$ Casi Siempre).

\section{Procedimiento}

Este estudio considera dos evaluaciones realizadas a las participantes: una primera aplicación inicial, y la evaluación correspondiente a un año de seguimiento desde esa primera evaluación. Todos los análisis se realizaron con el paquete estadístico SPSS 15.0, excepto el análisis factorial confirmatorio de la escala RRS, que se realizó con el paquete estadístico EQS 6.1 (Bentler, 2006).

\section{Resultados}

La Tabla 1 muestra los valores descriptivos de cada variable en ambas mediciones y las correlaciones que se observaron entre ellas.

Tabla 1. Media, desviación típica y correlaciones entre las variables depresión, ansiedad, reflexión, rumiación negativa ( $\left.\mathrm{T}_{1}\right)$ y depresión en el seguimiento $\left(\mathrm{T}_{2}\right)$.

\begin{tabular}{|c|c|c|c|c|c|c|c|}
\hline & 1 & 2 & 3 & 4 & 5 & M & DE \\
\hline 1: Depresión $T_{1}$ & - & $0,47 * * *$ & $0,30 * * *$ & $0,46 * * *$ & $0,45 * * *$ & 16,30 & 9,54 \\
\hline 2: Ansiedad $\mathrm{T}_{1}$ & & & $0,31 * * *$ & $0,46 * * *$ & $0,39 * * *$ & 12,86 & 9,02 \\
\hline 3: Reflexión & & & & $0,57 * * *$ & $0,30 * * *$ & 8,54 & 4,66 \\
\hline 4: Rumiación negativa $\mathrm{T}_{1}$ & & & & & $0,43 * * *$ & 10,32 & 4,98 \\
\hline 5: Depresión $T_{2}$ & & & & & & 13,06 & 10,23 \\
\hline
\end{tabular}

*: $\mathrm{p}<0,05 ; * *: \mathrm{p}<0,01 ; * * *: \mathrm{p}<0,001$.

Tabla 2. Regresión lineal múltiple jerárquica para la variable depresión en su evaluación de seguimiento a seis meses.

\begin{tabular}{|c|c|c|c|c|c|}
\hline & & B & $\mathrm{EE}$ & $\beta$ & $\mathrm{sr}^{2}$ \\
\hline \multirow[t]{3}{*}{ Paso 1} & Constante & 3,81 & 1,15 & & \\
\hline & Depresión $T_{1}$ & $0,37 * * *$ & 0,07 & 0,34 & 0,09 \\
\hline & Ansiedad $\mathrm{T}_{1}$ & $0,25 * * *$ & 0,07 & 0,22 & 0,04 \\
\hline \multicolumn{6}{|c|}{$R=0,49 * * * ; R^{2}=0,24\left(R_{\text {ajustado }}^{2}=0,23\right)$} \\
\hline \multirow[t]{6}{*}{ Paso 2} & Constante & 1,30 & 1,38 & & \\
\hline & Depresión $T_{1}$ & $0,29 * * *$ & 0,07 & 0,27 & 0,05 \\
\hline & Ansiedad $\mathrm{T}_{1}$ & $0,17 * *$ & 0,07 & 0,15 & 0,02 \\
\hline & Reflexión $\mathrm{T}_{1}$ & $-0,05^{*}$ & 0,14 & $-0,02$ & 0,00 \\
\hline & Rumiación negativa $T_{1}$ & $0,51 * *$ & 0,15 & 0,25 & 0,03 \\
\hline & \multicolumn{5}{|c|}{$R=0,53 ; R^{2}=0,28\left(R_{\text {ajustado }}^{2}=0,27\right)$} \\
\hline
\end{tabular}

$*: \mathrm{p}<0,05 ; * *: \mathrm{p}<0,01 ; * * *: \mathrm{p}<0,001$

Variable dependiente: Depresión $\mathrm{T}_{2}$

$B$ : Coeficiente de regresión no estandarizado, $E E$ : Error estándar, $\beta$ : Coeficiente de regresión estandarizado, $s r^{2}=$ Correlación semiparcial al cuadrado.

Nota: Este análisis fue repetido ingresando en el primer paso, como variable control, la inclusión de las participantes en los grupos experimentales o controles de un programa preventivo, la que no mostró significación, por lo que, para fines de claridad, no se la incluyó en el presente análisis. Dado que algunas variables no eran completamente normales, el análisis fue repetido con las variables normalizadas, lo que tampoco influyó en los resultados, por lo que se presentan los datos obtenidos con las variables originales. 
La Tabla 2 muestra los resultados de la regresión múltiple jerárquica implementada. En un primer paso se ingresó la medición inicial de depresión (BDI-II) y ansiedad (BAI) de modo de controlar el efecto de los valores iniciales de estas variables. Se decidió controlar tanto el efecto de la depresión inicial como de la ansiedad inicial, dada la estrecha interrelación entre ambas variables, y dado también que ambas han mostrado relación con rumiación. El coeficiente de regresión observado fue de $0,49(p<0,001)$. Ambas variables evaluadas mostraron una asociación significativa $(p<0,000)$ con la sintomatología depresiva evaluada un año después. En un segundo paso se ingresó reflexión y rumiación negativa. Se observó un incremento significativo de la varianza explicada $\left(\mathrm{R}^{2}=0,53, p<0,001\right)$. Sólo rumiación negativa mostró una asociación significativa con depresión posterior $(\beta=3,50, p<0,001)$. Reflexión mostró una relación negativa pero no significativa con depresión posterior ( $\beta=-0,02, p=0,75$ ). La última columna de la Tabla 2 permite observar los valores del coeficiente de correlación semiparcial de cada variable elevado al cuadrado. Este coeficiente permite analizar la contribución específica que cada predictor realiza a la explicación de la varianza total de la variable dependiente, cuando se parcializa en el predictor la varianza que comparte con los restantes predictores (Cohen, Cohen, West \& Aiken, 2003).

Se verificó el adecuado cumplimiento de los supuestos de la regresión. Para ello, se debió transformar algunas variables. Sin embargo, no se observaron diferencias en los resultados entre la ecuación con las variables transformadas y originales, por lo que sólo se informan las segundas.

\section{Discusión}

Los resultados obtenidos son convergentes con investigaciones recientes (Burwell, \& Shirk, 2007; Treynor et al., 2003) en mostrar que la rumiación negativa es un factor de riesgo para el desarrollo de sintomatología depresiva en adolescentes, y que la reflexión no tiene el mismo tipo de influencia.

Los estudios se han centrado fundamentalmente en la relación entre rumiación y depresión, sin embargo, recientes investigaciones han mostrado que la relación de la rumiación con la ansiedad es tanto o más intensa que con la depresión y que incluso, en algún grado, la ansiedad mediaría las relaciones observadas de la rumiación con esta última (Calmes \& Roberts, 2007). De allí la importancia de haber controlado los síntomas depresivos y ansiosos presentes en la primera evaluación. Esto, junto al carácter prospectivo de la investigación realizada, permite descartar que la relación observada entre la rumiación negativa y los síntomas depresivos sea sólo un correlato de los síntomas afectivos.
La presente investigación evitó también el solapamiento de los ítems de rumiación empleados con los indicadores de sintomatología depresiva. Se empleó una versión depurada de la RRS que no contiene ítems que se pueda considerar que se superponen con sintomatología depresiva y generar relaciones espúreas.

En consecuencia, la focalización repetitiva en el sentimiento negativo, las lamentaciones acerca de su ocurrencia y la autoevaluación negativa de sí mismo, propias de la rumiación negativa, se mostraron como un claro factor predictor del desarrollo de sintomatología depresiva en las adolescentes de sexo femenino que fueron parte de este estudio. Por el contrario, la reflexión acerca del malestar que se experimenta se mostró asociada sólo transversalmente a la presencia de sintomatología depresiva, pero no en forma prospectiva, sugiriendo que es una estrategia que se activa en momentos de malestar emocional, pero que no necesariamente contribuye a la aparición, mantención o agravamiento de éste. Incluso, se ha observado que podría ser una estrategia positiva, contribuyendo a una búsqueda de soluciones, a una mayor comprensión de lo vivido y a una elaboración de las experiencias, pero la investigación al respecto es todavía reducida (Treynor et al., 2003; Watkins, 2008). Sin embargo, las evidencias respecto del rol positivo o inocuo de la reflexión no son todas convergentes (NolenHoeksema et al., 2007; Miranda \& Nolen-Hoeksema, 2007), indicando que es necesario delimitar mejor este concepto para poder evaluar con mayor precisión sus efectos. Ello permitirá entender mejor, asimismo, la explicación de por qué reflexión y rumiación, tal como son evaluados por el RRS, se correlacionan entre sí tanto transversal como longitudinalmente.

Dado que hasta recientemente la mayoría de los investigadores han empleado el concepto de rumiación sin distinguir entre reflexión y rumiación negativa, es probable que la amplia evidencia respecto a que la rumiación constituye una estrategia de afrontamiento perjudicial, que tiene un rol en desencadenar, mantener y acentuar afectos disfóricos, sea atribuible, fundamentalmente, al rol de la rumiación negativa, pero todavía existen insuficientes datos al respecto (Abela \& Hankin, 2009; Nolen-Hoeksema, 2004). De comprobarse ello sería probablemente más clarificador que el concepto de rumiación se usara sólo para referirse a la rumiación negativa y se considerara la reflexión como una forma de pensamiento repetitivo distinta, con sus propias características.

Una limitación de la presente investigación es que se realizó sólo en mujeres y no es clara cuan generalizables son sus resultados a hombres ni a un grupo etario más amplio que el considerado. Con esas salvedades, los resultados obtenidos son indicadores de que un objetivo que los programas preventivos y de tratamiento de la depresión en adolescentes debieran considerar es el favorecimiento de estrategias de afrontamiento y regulación emocional 
adaptativas, evitando la rumiación negativa. Probablemente, programas que consideren este objetivo pueden ser una de las estrategias útiles para intervenir en la fuerte acentuación que el malestar emocional y los trastornos afectivos tienen a partir de la pubertad, especialmente, en el sexo femenino.

\section{Referencias}

Abela, J. R. Z. \& Hankins, B. (2009). Cognitive vulnerability to depression in adolescent: A developmental psychopathology perspective. En: Hoeksema, S. (Ed.), Handbook of Depression in Adolescents (pp. 335-376). New York: Routledge.

Beck A. T., \& Steer, R. A. (1993). BAI Beck Anxiety Inventory Manual. San Antonio, TX: The Psychological Corporation

Beck A. T., Steer, R. A., \& Brown, G. K. (1996). BDI-II. Beck Depression Inventory-Second Edition. Manual. San Antonio, TX: The Psychological Corporation.

Bentler, P. (2006). EQS 6 Structural Equations Program Manual. Encino, CA: Multivariate Software, Inc.

Burwell, R. A. \& Shirk, S. R. (2007). Subtypes of rumination in adolescence: Associations between brooding, reflection, depressive symptoms and coping. Journal of Clinical Child and Adolescent Psychology, 36, $56-65$.

Calmes, C. A. \& Roberts, J. E. (2007). Repetitive thought and emotional distress: Rumination and worry as prospective predictors of depressive and anxious symptomatology. Cognitive Therapy Research, 30, 343-356.

Cohen, J., Cohen, P., West, S. G. \& Aiken, L. S. (2003). Applied multiple regression/correlation analysis for the behavioral sciences (3rd ed.). New Jersey: Lawrence Erlbaum Associates.

Cova, F. \& Melipillán, R. (2007). Evaluación de sintomatología emocional $y$ de trastornos depresivos y ansiosos en adolescentes chilenos. Póster presentado en el VII Congreso Nacional de Psicología: Aportes y Desafíos, 9 y 10 de Noviembre, Santiago, Chile.

Cova, F., Rincón, P. \& Melipillán, R. (2007). Rumiación y presencia de sintomatología ansiosa y depresiva en adolescentes. Revista Mexicana de Psicología, 24, 175-183.

Hilt, L. \& Nolen-Hoeksema, S. (2009). The emergence of gender differences in depression in adolescence. En: S. Hoeksema (ed.), Handbook of depression in adolescents (pp. 111-135). New York: Routledge.

Kuyken, W., Watkins, E., Holden, E. \& Cook, W. (2006). Rumination in adolescents at risk for depression. Journal of Affective Disorders, 96, 39-47.

Lyubomirsky, S., \& Nolen-Hoeksema, S. (1995). Self-perpetuating propierties of dysphoric rumination. Journal of Personality and Social Psychology, 65, 176-190.

Lyubomirsky, S., Caldwell, N. D., \& Nolen-Hoeksema, S. (1998). Effects of self-focused ruminative and distracting responses to depressed mood on retrieval of autobiographical memories. Journal of Personality and Social Psychology, 75, 166-177.
Melipillán, R., Cova, F. \& Rincón, P. (2008). Propiedades psicométricas del Inventario de Depresión de Beck-II en adolescentes chilenos. Terapia Psicológica, 26(1), 59-69.

Miranda, R. \& Nolen-Hoeksema, S. (2007). Brooding and reflection: Rumination predicts suicidal ideation at 1-year follow-up in a community sample. Behaviour Research and Therapy, 45, 3088-3095.

Nolen-Hoeksema, S. (1991). Responses to depression and their effects on the duration of depressive episodes. Journal of Abnormal Psychology, 100, 569-582.

Nolen-Hoeksema, S. (2004). The Response Styles Theory. En C. Papageorgiou \& A. Wells (eds.), Depressive Ruminations: Nature, theory, and treatment (p. 107-123). West Sussex: Wiley \& Sons.

Nolen-Hoeksema, S., Stice, E., Wade, E. \& Bohon, C. (2007). Reciprocal relations between rumination and bulimic, substance abuse, and depressive symptoms in female adolescents. Journal of Abnormal Psychology, 116, 198-207.

Papageorgiou, C., \& Siegle, G. J. (2003). Rumination and depression: Advances in theory and research. Cognitive Therapy and Research, $27,243-245$

Papageorgiou, C. \& Wells, A. (2004) Nature, functions, and beliefs about depressive rumination. En C. Papageorgiou \& A. Wells (eds.), Depressive Ruminations: Nature, theory, and treatment (pp. 3-20). West Sussex: Wiley \& Sons.

Piotrowski, C. (1999). The status of the Beck Anxiety Inventory in contemporary research. Psychological Reports, 85, 261-262.

Sanz, J. \& Navarro, M. E. (2003). Propiedades psicométricas de una versión española del Inventario de Ansiedad de Beck (BAI) en estudiantes universitarios. Ansiedad y Estrés, 9, 59-84.

Sanz, J., Navarro, M. E. \& Vázquez, C. (2003). Adaptación española del inventario para la depresión de Beck-II (BDI-II): 1. Propiedades psicométricas en estudiantes universitarios. Análisis y Modificación de Conducta, 29, 239-288.

Thomsen, D. (2006). The association between rumination and negative affect: A review. Cognition and Emotion, 20, 1216-1235.

Treynor, W., González, R., \& Nolen-Hoeksema, S. (2003). Rumiation reconsidered: A psychometric analysis. Cognitive Therapy and Research, 27, 247-259.

Watkins, E. (2004). Adaptive and maladaptive ruminative self-focus during emotional processing. Behavior Research and Therapy, 42, $1037-1052$.

Watkins, E. (2008). Constructive and unconstructive repetitive thought. Psychological Bulletin, 134, 163-206.

Whisman, M, Pérez J., \& Ramel, W. (2000). Factor structure of the Beck Depression Inventory-Second Edition (BDI-II) in a student sample. Journal of Clinical Psychology, 56, 545-551.

Zahn-Waxler, C., Crick, N., Shirtcliff, E. \& Woods, K. (2006). The origin and development of psychopathology in females and males. En: D. Cichetti \& D. Cohen (Eds.), Developmental Psychopathology (v. 1, pp. 76-138). New Jersey: Wiley \& Sons. 